\title{
Global Solutions of Certain Second-Order Differential Equations with a High Degree of Apparent Singularity ${ }^{\star}$
}

\author{
Ryu $S A S A K I^{\dagger}$ and Kouichi TAKEMURA $\ddagger$ \\ $\dagger$ Yukawa Institute for Theoretical Physics, Kyoto University, Kyoto 606-8502, Japan \\ E-mail: ryu@yukawa.kyoto-u.ac.jp \\ $\ddagger$ Department of Mathematics, Faculty of Science and Technology, Chuo University, \\ 1-13-27 Kasuga, Bunkyo-ku Tokyo 112-8551, Japan \\ E-mail: takemura@math.chuo-u.ac.jp
}

Received July 24, 2012, in final form November 07, 2012; Published online November 15, 2012 http://dx.doi.org/10.3842/SIGMA.2012.085

\begin{abstract}
Infinitely many explicit solutions of certain second-order differential equations with an apparent singularity of characteristic exponent -2 are constructed by adjusting the parameter of the multi-indexed Laguerre polynomials.
\end{abstract}

Key words: multi-indexed orthogonal polynomials; solvable systems; Fuchsian differential equations; Heun's equation; apparent singularities; high characteristic exponents

2010 Mathematics Subject Classification: 33C45; 33C47

\section{Introduction}

In recent years, global solutions of Fuchsian differential equations with more than three regular singularities and those of the differential equations of confluent types, attracted attention $[11,29]$. The multi-indexed Laguerre and Jacobi polynomials $[10,19]^{1}$ and their simplest (one-indexed) cases, i.e. the exceptional orthogonal polynomials, provide explicit examples of infinitely many global solutions of such equations. They are obtained by Darboux-Crum transformations [1, 3, 4, 14] applied to the exactly solvable Schrödinger equations [2, 13] of the radial oscillator potential and the Pöschl-Teller potential. In these global solutions, all the extra singularities (i.e. those added by Darboux-Crum transformations) turn out to be apparent and the characteristic exponents are all -1 . This is a generic property of the Darboux-Crum transformations. The role of Darboux transformations in iso-monodromic deformations has been known for some time [5] and some explicit examples of monodromy free rational potentials were given in $[8,15]$.

In the present paper, we construct, by adjusting the parameter of the multi-indexed Laguerre polynomials, several families of infinitely many global solutions of Schrödinger equations with two or more finite regular singularities and one irregular singularity at infinity. At one of the regular singular points, these solutions have an apparent singularity of characteristic exponent -2 . We now show one of the examples which is labeled as (E) in Section 3. The Hamiltonian is given by

$$
\mathcal{H}=-\frac{d^{2}}{d x^{2}}+x^{2}+\frac{195}{4 x^{2}}-16-2 \frac{d^{2} \log \left(x^{2}+6\right)^{3}\left(x^{2}+14\right)}{d x^{2}} .
$$

*This paper is a contribution to the Special Issue "Superintegrability, Exact Solvability, and Special Functions". The full collection is available at http://www.emis.de/journals/SIGMA/SESSF2012.html

${ }^{1}$ For the bibliography of various exceptional orthogonal polynomials see references in [19]. 
We change the variable by $\eta=x^{2}$. Then the equation $\mathcal{H} \psi(x)=\mathcal{E} \psi(x)(\mathcal{E} \in \mathbb{C})$ in the variable $\eta$ has apparent singularities $\eta=-6$ and $\eta=-14$, whose characteristic exponent is -2 and -1 respectively. All the apparent singularities are located outside of the half line $(0, \infty)$, thus the eigenfunctions of the Schrödinger equations are constructed by using Darboux-Crum transformation, and they are global solutions of the differential equations. The essential part of the numerators of these global solutions form orthogonal polynomials over a half line $(0, \infty)$, with the square of the denominator forming the part of the weight functions $e^{-x} x^{7} /\left\{(x+6)^{4}(x+14)^{2}\right\}$. The polynomials in the numerators of the global solutions are obtained within the framework of multi-indexed orthogonal polynomials, and their degrees have "gaps". In this example, the polynomials of degrees 0,1 , and 2 are missing. We also report examples (A), (B), (C), (D), $(\mathrm{F}),(\mathrm{G})$ in the two index case and an example in the three index case. Each example has its peculiar properties (see section three). The orthogonal polynomials related to (A) and (B) are also reported in [9].

This paper is organised as follows. In Section 2, we review the Darboux transformations with the induced singularities. In Section 3 we show that the special cases of multi-indexed Laguerre polynomials provide several families of infinitely many global solutions of confluent differential equations with two or more finite regular singularities and one irregular singularity at infinity. They have an apparent singularity of characteristic exponent -2 . The final section is for a summary and comments.

\section{Darboux transformations and induced singularities}

Our starting point is a generic Schrödinger equation with a Hamiltonian $\mathcal{H}$

$$
\mathcal{H}=-\frac{d^{2}}{d x^{2}}+U(x),
$$

in which $U(x)$ is a meromorphic potential. Let $\psi(x)$ and $\varphi(x)$ be two distinct solutions, not necessarily square-integrable eigenfunctions, of the Schrödinger equation

$$
\mathcal{H} \psi(x)=\mathcal{E} \psi(x), \quad \mathcal{H} \varphi(x)=\tilde{\mathcal{E}} \varphi(x), \quad \mathcal{E}, \tilde{\mathcal{E}} \in \mathbb{C} .
$$

Then it is elementary to show that a new function

$$
\psi^{(1)}(x) \stackrel{\text { def }}{=} \frac{\mathrm{W}[\varphi, \psi](x)}{\varphi(x)}=\frac{\varphi(x) \psi^{\prime}(x)-\varphi^{\prime}(x) \psi(x)}{\varphi(x)}
$$

is a solution of a new Schrödinger equation with a deformed Hamiltonian $\mathcal{H}^{(1)}$

$$
\mathcal{H}^{(1)}=-\frac{d^{2}}{d x^{2}}+U^{(1)}(x), \quad U^{(1)}(x) \stackrel{\text { def }}{=} U(x)-2 \frac{d^{2} \log \varphi(x)}{d x^{2}},
$$

with the same energy $\mathcal{E}$

$$
\mathcal{H}^{(1)} \psi^{(1)}(x)=\mathcal{E} \psi^{(1)}(x) .
$$

The zeros of the "seed" solution $\varphi(x)$ produce singularities of the equation, and there are no multiplicities of the zeros of $\varphi(x)$ except for the singularities of $U(x)$, because the function $\varphi(x)$ satisfies the Schrödinger equation and the exponents at a holomorphic point are 0 or 1 .

To obtain the deformed Hamiltonian which admits an apparent singularity of exponent -2 or higher, we use Darboux-Crum transformations $[1,3,4,14]$. Let $\left\{\varphi_{j}(x), \tilde{\mathcal{E}}_{j}\right\}, j=1, \ldots, M$ be distinct seed solutions of the original Schrödinger equation (2.1) and as above $\psi(x)$ be a solution with the energy $\mathcal{E}$

$$
\mathcal{H} \psi(x)=\mathcal{E} \psi(x), \quad \mathcal{H} \varphi_{j}(x)=\tilde{\mathcal{E}}_{j} \varphi_{j}(x), \quad \mathcal{E}, \tilde{\mathcal{E}}_{j} \in \mathbb{C}, \quad j=1, \ldots, M .
$$


By repeating the above Darboux transformation $M$-times, we obtain a new function

$$
\psi^{(M)}(x) \stackrel{\text { def }}{=} \frac{\mathrm{W}\left[\varphi_{1}, \ldots, \varphi_{M}, \psi\right](x)}{\mathrm{W}\left[\varphi_{1}, \ldots, \varphi_{M}\right](x)},
$$

which satisfies an $M$-th deformed Schrödinger equation with the same energy $[1,3,14]$

$$
\begin{aligned}
& \mathcal{H}^{(M)} \psi^{(M)}(x)=\mathcal{E} \psi^{(M)}(x), \\
& \mathcal{H}^{(M)}=-\frac{d^{2}}{d x^{2}}+U^{(M)}(x), \quad U^{(M)}(x) \stackrel{\text { def }}{=} U(x)-2 \frac{d^{2} \log \mathrm{W}\left[\varphi_{1}, \ldots, \varphi_{M}\right](x)}{d x^{2}}, \\
& \left(\psi^{(M)}, \psi^{(M)}\right)=\prod_{j=1}^{M}\left(\mathcal{E}-\tilde{\mathcal{E}}_{j}\right) \cdot(\psi, \psi) .
\end{aligned}
$$

Here $\mathrm{W}\left[f_{1}, \ldots, f_{N}\right](x)$ is a Wronskian

$$
\mathrm{W}\left[f_{1}, \ldots, f_{N}\right](x) \stackrel{\text { def }}{=} \operatorname{Det}\left(\frac{d^{k-1} f_{j}(x)}{d x^{k-1}}\right)_{1 \leq j, k \leq N},
$$

and the inner product among real functions is defined as usual

$$
(f, g) \stackrel{\text { def }}{=} \int f(x) g(x) d x \text {. }
$$

Except for the singularities of the potential, a zero of the Wronskian $\mathrm{W}\left[\varphi_{1}, \ldots, \varphi_{M}\right](x)$

$$
\mathrm{W}\left[\varphi_{1}, \ldots, \varphi_{M}\right](x)=c_{0}\left(x-x_{0}\right)^{m}+O\left(x-x_{0}\right)^{m+1}, \quad m \in \mathbb{Z}_{>0},
$$

corresponds to an apparent singular point of the deformed Hamiltonian $\mathcal{H}^{(M)}$

$$
x \approx x_{0}, \quad U^{(M)}(x)=U\left(x_{0}\right)+\frac{2 m}{\left(x-x_{0}\right)^{2}}+\text { regular terms. }
$$

The characteristic exponents of the solutions of the new Schrödinger equation (2.2) at the apparent singularity $x_{0}$ are determined by

$$
\rho(\rho-1)-2 m=0 .
$$

For certain values of the integer $m$, the monodromy of the added singularity becomes trivial

$$
\begin{array}{ll}
m=1, & \rho=-1,2, \\
m=3, & \rho=-2,3, \\
m=6, & \rho=-3,4, \quad \text { etc. }
\end{array}
$$

When all the added singularities are of trivial monodromy, the possibility of global solutions arises. The infinite families of the exceptional (or one-indexed) Laguerre and Jacobi polynomials [19] are the examples of the global solutions of second-order Fuchsian (and its confluent limit) equations with many extra singularities with all $\rho=-1$. This is the generic case, since any solution $\varphi(x)$ of the Schrödinger equation (2.1) with the radial oscillator potential (3.1) or the Pöschl-Teller potential [19] cannot have a multiple zero $(m \geq 2)$.

\section{Multi-indexed Laguerre polynomials}

In this section, we present several families of infinitely many global solutions with a high degree $(\rho=-2)$ of apparent singularity. The strategy is simple. We start from certain "nongeneric" multi-indexed Laguerre polynomials and choose the parameter $g$ such that a triple zero is achieved for the seed solution (the Wronskian). Then we construct the global solutions explicitly. When the zeros are outside the half line $(0, \infty)$, the global solutions are square integrable and they are eigenfunctions of the deformed Hamiltonian. 


\section{1 "Non-generic" multi-indexed Laguerre polynomials}

For the multi-indexed Laguerre polynomials [19], we adopt the Schrödinger equation (2.1) with the radial oscillator potential $[2,13]$

$$
U(x ; g)=x^{2}+\frac{g(g-1)}{x^{2}}-(1+2 g), \quad g>0, \quad 0<x<\infty .
$$

It has a regular singularity at $x=0$ with the characteristic exponents $\rho=g, 1-g$ and an irregular singularity at $x=\infty$. The eigenpolynomial solutions are

$$
\begin{aligned}
& \mathcal{H} \phi_{n}(x ; g)=\mathcal{E}_{n} \phi_{n}(x ; g), \quad \mathcal{E}_{n}=4 n, \\
& \phi_{n}(x ; g)=e^{-x^{2} / 2} x^{g} L_{n}^{\left(g-\frac{1}{2}\right)}(\eta(x)), \quad \eta(x) \stackrel{\text { def }}{=} x^{2}, \quad n=0,1, \ldots,
\end{aligned}
$$

in which $L_{n}^{(\alpha)}(\eta)$ is the degree $n$ Laguerre polynomial in $\eta$. They form a complete set of orthogonal polynomials over $(0, \infty)$

$$
\begin{aligned}
& \left(\phi_{n}, \phi_{m}\right) \stackrel{\text { def }}{=} \int_{0}^{\infty} \phi_{n}(x ; g) \phi_{m}(x ; g) d x=\frac{1}{2} \int_{0}^{\infty} e^{-\eta} \eta^{g-\frac{1}{2}} L_{n}^{\left(g-\frac{1}{2}\right)}(\eta) L_{m}^{\left(g-\frac{1}{2}\right)}(\eta) d \eta=h_{n}(g) \delta_{n m}, \\
& h_{n}(g) \stackrel{\text { def }}{=} \frac{1}{2 n !} \Gamma\left(n+g+\frac{1}{2}\right) .
\end{aligned}
$$

The generic multi-indexed Laguerre polynomials are constructed by the Darboux-Crum transformations (2.3) in terms of two types of polynomial $\left(\mathrm{v} \in \mathbb{Z}_{>0}\right)$ seed solutions, called the virtual state solutions [19],

$$
\begin{array}{lll}
\tilde{\phi}_{\mathrm{v}}^{\mathrm{I}}(x ; g) \stackrel{\text { def }}{=} e^{\frac{1}{2} x^{2}} x^{g} L_{\mathrm{v}}^{\left(g-\frac{1}{2}\right)}(-\eta(x)), & \tilde{\mathcal{E}}_{\mathrm{v}}^{\mathrm{I}}(g) \stackrel{\text { def }}{=}-4\left(g+\mathrm{v}+\frac{1}{2}\right), & \left(\tilde{\phi}_{\mathrm{v}}^{\mathrm{I}}, \tilde{\phi}_{\mathrm{v}}^{\mathrm{I}}\right)=\infty, \\
\tilde{\phi}_{\mathrm{v}}^{\mathrm{II}}(x ; g) \stackrel{\text { def }}{=} e^{-\frac{1}{2} x^{2}} x^{1-g} L_{\mathrm{v}}^{\left(\frac{1}{2}-g\right)}(\eta(x)), & \tilde{\mathcal{E}}_{\mathrm{v}}^{\mathrm{II}}(g) \stackrel{\text { def }}{=}-4\left(g-\mathrm{v}-\frac{1}{2}\right), \quad\left(\tilde{\phi}_{\mathrm{v}}^{\mathrm{II}}, \tilde{\phi}_{\mathrm{v}}^{\mathrm{II}}\right)=\infty,
\end{array}
$$

with certain constraints on the range of the parameter $g$. In order to generate a wider class of global solutions with a high degree of apparent singularities, we will adopt non-virtual state polynomial seed solutions, too:

$$
\tilde{\phi}_{\mathrm{v}}^{\mathrm{III}}(x ; g) \stackrel{\text { def }}{=} e^{\frac{1}{2} x^{2}} x^{1-g} L_{\mathrm{v}}^{\left(\frac{1}{2}-g\right)}(-\eta(x)), \quad \tilde{\mathcal{E}}_{\mathrm{v}}^{\mathrm{III}}(g) \stackrel{\text { def }}{=}-4(\mathrm{v}+1), \quad\left(\tilde{\phi}_{\mathrm{v}}^{\mathrm{III}}, \tilde{\phi}_{\mathrm{v}}^{\mathrm{III}}\right)=\infty .
$$

Here we summarise the definition of "non-generic" multi-indexed Laguerre polynomials.

Definition. The "non-generic" multi-indexed Laguerre polynomials are the main part of the eigenfunctions $\psi^{(M)}(x)(2.3)$ generated by the multiple Darboux transformations with the eigenfunctions $\left\{\phi_{n}(x ; g)\right\}$ and the three types of seed solutions (3.3), (3.4) and (3.5), the virtual and non-virtual state solutions, and that the parameter $g$ is restricted only by the positivity $g>0$.

The regularity of the solutions and/or the positivity of the resultant weight functions etc. must be verified in each specific case of the chosen parameter values. This is in good contrast with the multi-indexed Laguerre and Jacobi polynomials defined in [19], in which the parameters are restricted (equations (23) and (24) of [11]) so that the seed solutions are of definite sign and their inverse are not square integrable

$$
\left(1 / \tilde{\phi}_{\mathrm{v}}^{\mathrm{I}}, 1 / \tilde{\phi}_{\mathrm{v}}^{\mathrm{I}}\right),=\left(1 / \tilde{\phi}_{\mathrm{v}}^{\mathrm{II}}, 1 / \tilde{\phi}_{\mathrm{v}}^{\mathrm{II}}\right)=\infty .
$$

These two additional properties guarantee the regularity of the solutions and the positivity of the weight function. In other words, these restrictions are lifted for the possible construction of the non-generic multi-indexed Laguerre polynomials. In the singular solution cases, the square integrability is irrelevant and we also consider $g<0$ example $(\mathrm{F})$.

For the $M$-index case, the seed solutions is $\mathrm{W}\left[\varphi_{\mathrm{v}_{1}}, \ldots, \varphi_{\mathrm{v}_{M}}\right](x)$. We look for the possibility that this function has triple or higher zeros at some points. 


\subsection{Two-index case}

Here we consider the simplest multi-index case, the two-index case

$$
\phi_{n}^{(2)}(x) \stackrel{\text { def }}{=} \frac{\mathrm{W}\left[\varphi_{1}, \varphi_{2}, \phi_{n}\right](x)}{\mathrm{W}\left[\varphi_{1}, \varphi_{2}\right](x)} .
$$

We focus on the seed solutions of the form

$$
\mathrm{W}\left[\tilde{\phi}_{\mathrm{v}_{1}}^{\mathrm{III}}(x ; g), \tilde{\phi}_{\mathrm{v}_{2}}^{\mathrm{I}}(x ; g)\right](x), \quad \mathrm{W}\left[\tilde{\phi}_{\mathrm{v}_{1}}^{\mathrm{III}}(x ; g), \tilde{\phi}_{\mathrm{v}_{2}}^{\mathrm{II}}(x ; g)\right](x), \quad \mathrm{W}\left[\tilde{\phi}_{\mathrm{v}_{1}}^{\mathrm{I}}(x ; g), \tilde{\phi}_{\mathrm{v}_{2}}^{\mathrm{II}}(x ; g)\right](x),
$$

with lower degrees $\mathrm{v}_{1}$ and $\mathrm{v}_{2}$ and evaluate the discriminants of the polynomial part of the seed solutions. At the roots of the discriminant, which is a polynomial in $g$, the above seed solution has a multiple zero. The first two are the non-generic type and the last one is the generic type. As we will show in some detail, they have different features.

So far we have encountered the following seven cases:
$(A): \mathrm{W}\left[\tilde{\phi}_{1}^{\mathrm{III}}\left(x ; \frac{3}{4}\right), \tilde{\phi}_{2}^{\mathrm{I}}\left(x ; \frac{3}{4}\right)\right](x)$,
$(B): \mathrm{W}\left[\tilde{\phi}_{2}^{\mathrm{III}}\left(x ; \frac{1}{4}\right), \tilde{\phi}_{1}^{\mathrm{I}}\left(x ; \frac{1}{4}\right)\right](x)$,
$(C): \mathrm{W}\left[\tilde{\phi}_{2}^{\mathrm{III}}\left(x ; \frac{9}{4}\right), \tilde{\phi}_{1}^{\mathrm{II}}\left(x ; \frac{9}{4}\right)\right](x)$,
$(D): \mathrm{W}\left[\tilde{\phi}_{1}^{\mathrm{III}}\left(x ; \frac{9}{4}\right), \tilde{\phi}_{2}^{\mathrm{II}}\left(x ; \frac{9}{4}\right)\right](x)$,
$(E): \mathrm{W}\left[\tilde{\phi}_{2}^{\mathrm{I}}\left(x ; \frac{15}{2}\right), \tilde{\phi}_{1}^{\mathrm{II}}\left(x ; \frac{15}{2}\right)\right](x)$,
$(F): \mathrm{W}\left[\tilde{\phi}_{1}^{\mathrm{I}}\left(x ;-\frac{13}{2}\right), \tilde{\phi}_{2}^{\mathrm{II}}\left(x ;-\frac{13}{2}\right)\right](x)$,
$(G): \mathrm{W}\left[\tilde{\phi}_{3}^{\mathrm{I}}\left(x ; \frac{53}{2}\right), \tilde{\phi}_{1}^{\mathrm{II}}\left(x ; \frac{53}{2}\right)\right](x)$.

\subsubsection{Seed solution case (A)}

In this case

$$
\begin{aligned}
& \mathrm{W}\left[\tilde{\phi}_{1}^{\mathrm{III}}(x ; g), \tilde{\phi}_{2}^{\mathrm{I}}(x ; g)\right](x) \\
& \quad=\frac{1}{16}(2 g+1) e^{x^{2}}\left(-9+18 g+4 g^{2}-8 g^{3}+\left(18-8 g^{2}\right) x^{2}+(12+8 g) x^{4}+8 x^{6}\right) .
\end{aligned}
$$

The discriminant of the polynomial in the variable $\eta\left(=x^{2}\right)$ is

$$
2048(2 g-3)(2 g+3)^{2}(4 g-3)^{2},
$$

and $g=\frac{3}{4}$ gives a cubic zero

$$
\mathrm{W}\left[\tilde{\phi}_{1}^{\mathrm{III}}\left(x ; \frac{3}{4}\right), \tilde{\phi}_{2}^{\mathrm{I}}\left(x ; \frac{3}{4}\right)\right](x)=\frac{5}{256} e^{x^{2}}\left(3+4 x^{2}\right)^{3} .
$$

The potential is

$$
U_{A}(x)=x^{2}-\frac{3}{16 x^{2}}+\frac{48}{3+4 x^{2}}-\frac{288}{\left(3+4 x^{2}\right)^{2}}-\frac{13}{2} .
$$

\subsubsection{Seed solution case (B)}

In this case

$$
\begin{aligned}
& \mathrm{W}\left[\tilde{\phi}_{2}^{\mathrm{III}}(x ; g), \tilde{\phi}_{1}^{\mathrm{I}}(x ; g)\right](x) \\
& \quad=\frac{1}{16}(2 g-3) e^{x^{2}}\left(5-2 g-20 g^{2}+8 g^{3}+\left(10+16 g-8 g^{2}\right) x^{2}+(20-8 g) x^{4}+8 x^{6}\right) .
\end{aligned}
$$

The discriminant of the polynomial in $\eta$ is

$$
-2048(2 g-5)^{2}(2 g+1)(4 g-1)^{2},
$$

and $g=\frac{1}{4}$ gives a cubic zero

$$
\mathrm{W}\left[\tilde{\phi}_{2}^{\mathrm{III}}\left(x ; \frac{1}{4}\right), \tilde{\phi}_{1}^{\mathrm{I}}\left(x ; \frac{1}{4}\right)\right](x)=-\frac{5}{256} e^{x^{2}}\left(3+4 x^{2}\right)^{3} .
$$

The potential is the same as $U_{A}(x)$ up to an additive constant

$$
U_{B}(x)=U_{A}(x)+1=x^{2}-\frac{3}{16 x^{2}}+\frac{48}{3+4 x^{2}}-\frac{288}{\left(3+4 x^{2}\right)^{2}}-\frac{11}{2} .
$$




\subsubsection{Seed solution case $(\mathrm{C})$}

In this case

$$
\begin{aligned}
& \mathrm{W}\left[\tilde{\phi}_{2}^{\mathrm{III}}(x ; g), \tilde{\phi}_{1}^{\mathrm{II}}(x ; g)\right](x) \\
& =\frac{1}{8} x^{3-2 g}\left(-135+174 g-68 g^{2}+8 g^{3}+\left(-54+48 g-8 g^{2}\right) x^{2}+(36-8 g) x^{4}+8 x^{6}\right) .
\end{aligned}
$$

The discriminant of the polynomial in $\eta$ is

$$
-2048(2 g-9)^{2}(2 g-3)(4 g-9)^{2},
$$

and $g=\frac{9}{4}$ gives a cubic zero

$$
\mathrm{W}\left[\tilde{\phi}_{2}^{\mathrm{III}}\left(x ; \frac{9}{4}\right), \tilde{\phi}_{1}^{\mathrm{II}}\left(x ; \frac{9}{4}\right)\right](x)=\frac{\left(3+4 x^{2}\right)^{3}}{64 x^{3 / 2}} .
$$

The potential is exactly the same as $U_{B}$

$$
U_{C}(x)=U_{B}(x)=x^{2}-\frac{3}{16 x^{2}}+\frac{48}{3+4 x^{2}}-\frac{288}{\left(3+4 x^{2}\right)^{2}}-\frac{11}{2} .
$$

Since the eigenvalue $\mathcal{E}_{n}=4 n$ is independent of $g$, the corresponding solutions $\phi_{n}^{(2)}(x)$ (3.6) for (B) and (C) satisfy the same equations. Thus they are identical. We will not consider case (C) in the next subsection.

\subsubsection{Seed solution case (D)}

In this case

$$
\begin{aligned}
& \mathrm{W}\left[\tilde{\phi}_{1}^{\mathrm{III}}(x ; g), \tilde{\phi}_{2}^{\mathrm{II}}(x ; g)\right](x) \\
& \quad=\frac{1}{8} x^{3-2 g}\left(-135+174 g-68 g^{2}+8 g^{3}+\left(54-48 g+8 g^{2}\right) x^{2}+(36-8 g) x^{4}-8 x^{6}\right) .
\end{aligned}
$$

The polynomial part is obtained from that of (3.9) by the change $x^{2} \rightarrow-x^{2}$. Thus the discriminant is the same and $g=\frac{9}{4}$ gives a cubic zero

$$
\mathrm{W}\left[\tilde{\phi}_{1}^{\mathrm{III}}\left(x ; \frac{9}{4}\right), \tilde{\phi}_{2}^{\mathrm{II}}\left(x ; \frac{9}{4}\right)\right](x)=-\frac{\left(-3+4 x^{2}\right)^{3}}{64 x^{3 / 2}} \text {. }
$$

The potential has a singularity in $(0, \infty)$

$$
U_{D}(x)=x^{2}-\frac{3}{16 x^{2}}+\frac{48}{-3+4 x^{2}}+\frac{288}{\left(-3+4 x^{2}\right)^{2}}-\frac{11}{2} .
$$

The corresponding solutions (3.6) are not square integrable and this sequence is not that of orthogonal polynomials.

Cases (E)-(G) belong to the generic type.

\subsubsection{Seed solution case $(\mathrm{E})$}

In this case

$$
\begin{aligned}
& \mathrm{W}\left[\tilde{\phi}_{2}^{\mathrm{I}}(x ; g), \tilde{\phi}_{1}^{\mathrm{II}}(x ; g)\right](x) \\
& =-\frac{9}{16}+\frac{5}{2} g^{2}-g^{4}+\left(\frac{9}{2}+9 g-2 g^{2}-4 g^{3}\right) x^{2}+\left(\frac{15}{2}-4 g-6 g^{2}\right) x^{4}-2(1+2 g) x^{6}-x^{8} .
\end{aligned}
$$


The discriminant of the polynomial in $\eta$ is

$$
-16(2 g-15)^{2}(2 g-3)(2 g+1)(2 g+3)^{2}
$$

and $g=\frac{15}{2}$ gives a cubic zero

$$
\mathrm{W}\left[\tilde{\phi}_{2}^{\mathrm{I}}\left(x ; \frac{15}{2}\right), \tilde{\phi}_{1}^{\mathrm{II}}\left(x ; \frac{15}{2}\right)\right](x)=\left(6+x^{2}\right)^{3}\left(14+x^{2}\right) .
$$

The potential is

$$
U_{E}(x)=x^{2}+\frac{195}{4 x^{2}}-\frac{144}{\left(6+x^{2}\right)^{2}}+\frac{12}{6+x^{2}}-\frac{112}{\left(14+x^{2}\right)^{2}}+\frac{4}{14+x^{2}}-16 .
$$

\subsubsection{Seed solution case $(F)$}

In this case

$$
\begin{aligned}
& \mathrm{W}\left[\tilde{\phi}_{1}^{\mathrm{I}}(x ; g), \tilde{\phi}_{2}^{\mathrm{II}}(x ; g)\right](x)=\frac{15}{16}-g-\frac{7}{2} g^{2}+4 g^{3}-g^{4}-\left(\frac{15}{2}+7 g-14 g^{2}+4 g^{3}\right) x^{2} \\
& \quad-\left(\frac{5}{2}-16 g+6 g^{2}\right) x^{4}+(6-4 g) x^{6}-x^{8} .
\end{aligned}
$$

The discriminant of the polynomial in $\eta$ is

$$
-16(2 g-5)^{2}(2 g-3)(2 g+1)(2 g+13)^{2}
$$

and at a negative value of the parameter $g=-\frac{13}{2}$ it gives a cubic zero

$$
\mathrm{W}\left[\tilde{\phi}_{1}^{\mathrm{I}}\left(x ;-\frac{13}{2}\right), \tilde{\phi}_{2}^{\mathrm{II}}\left(x ;-\frac{13}{2}\right)\right](x)=-\left(x^{2}-14\right)\left(x^{2}-6\right)^{3} .
$$

The potential is singular in $(0, \infty)$

$$
U_{F}(x)=x^{2}+\frac{195}{4 x^{2}}+\frac{144}{\left(x^{2}-6\right)^{2}}+\frac{12}{x^{2}-6}+\frac{112}{\left(x^{2}-14\right)^{2}}+\frac{4}{x^{2}-14}+12 .
$$

\subsubsection{Seed solution case $(G)$}

In this case

$$
\begin{aligned}
& \mathrm{W}\left[\tilde{\phi}_{3}^{\mathrm{I}}(x ; g), \tilde{\phi}_{1}^{\mathrm{II}}(x ; g)\right](x)=\frac{1}{96}\{(-3+2 g)(-1+2 g)(1+2 g)(3+2 g)(5+2 g) \\
& \quad+10(-3+2 g)(1+2 g)(3+2 g)(5+2 g) x^{2}+80(-1+g)(3+2 g)(5+2 g) x^{4} \\
& \left.\quad+160 g(5+2 g) x^{6}+80(3+2 g) x^{8}+32 x^{10}\right\} .
\end{aligned}
$$

The discriminant in $\eta$ is proportional to

$$
(2 g-3)(2 g+1)(2 g+3)^{2}(2 g+5)^{3}(10 g-39)^{2}
$$

and $g=\frac{39}{10}$ gives a cubic zero

$$
\mathrm{W}\left[\tilde{\phi}_{3}^{\mathrm{I}}\left(x ; \frac{39}{10}\right), \tilde{\phi}_{1}^{\mathrm{II}}\left(x ; \frac{39}{10}\right)\right](x)=\frac{1}{9375}\left(12+5 x^{2}\right)^{3}\left(2244+495 x^{2}+25 x^{4}\right) .
$$

The potential is

$$
\begin{aligned}
U_{G}(x)= & x^{2}+\frac{1131}{100 x^{2}}-\frac{44}{5}-\frac{1440}{\left(12+5 x^{2}\right)^{2}}+\frac{60}{12+5 x^{2}} \\
& +\frac{165000 x^{2}}{\left(2244+495 x^{2}+25 x^{4}\right)^{2}}+\frac{20\left(10 x^{2}-99\right)}{2244+495 x^{2}+25 x^{4}} .
\end{aligned}
$$

The basic structure of $(\mathrm{G})$ is essentially the same as that of $(\mathrm{E})$. So we will not present the explicit formulas of $(\mathrm{G})$, since they tend to be rather lengthy. 


\subsubsection{The detailed structure of these seed solutions}

In the non-generic cases $(\mathrm{A})$ and $(\mathrm{B})$, the potential functions are essentially the same, but the main eigenfunctions carry different coupling constants, $\left\{\phi_{n}\left(x ; \frac{3}{4}\right)\right\}$ and $\left\{\phi_{n}\left(x ; \frac{1}{4}\right)\right\}$. Thus the corresponding multi-indexed polynomials are entirely different. The equality (up to a sign) of the seed solutions of (A) and (B) is easily understood, when one notes the trivial identity

$$
\tilde{\phi}_{\mathrm{v}}^{\mathrm{I}}(x ; g)=\tilde{\phi}_{\mathrm{v}}^{\mathrm{III}}(x ; 1-g) .
$$

In fact, we have

$$
\begin{aligned}
& \tilde{\phi}_{1}^{\mathrm{I}}\left(x ; \frac{1}{4}\right)=\tilde{\phi}_{1}^{\mathrm{III}}\left(x ; \frac{3}{4}\right)=\frac{1}{4} e^{x^{2} / 2} x^{1 / 4}\left(3+4 x^{2}\right), \\
& \left(1 / \tilde{\phi}_{1}^{\mathrm{I}}\left(x ; \frac{1}{4}\right), 1 / \tilde{\phi}_{1}^{\mathrm{I}}\left(x ; \frac{1}{4}\right)\right)=\left(1 / \tilde{\phi}_{1}^{\mathrm{III}}\left(x ; \frac{3}{4}\right), 1 / \tilde{\phi}_{1}^{\mathrm{III}}\left(x ; \frac{3}{4}\right)\right)<\infty, \\
& \tilde{\phi}_{2}^{\mathrm{I}}\left(x ; \frac{3}{4}\right)=\tilde{\phi}_{2}^{\mathrm{III}}\left(x ; \frac{1}{4}\right)=\frac{1}{32} e^{x^{2} / 2} x^{3 / 4}\left(3+4 x^{2}\right)\left(15+4 x^{2}\right), \\
& \left(1 / \tilde{\phi}_{2}^{\mathrm{I}}\left(x ; \frac{3}{4}\right), 1 / \tilde{\phi}_{2}^{\mathrm{I}}\left(x ; \frac{3}{4}\right)\right)=\left(1 / \tilde{\phi}_{2}^{\mathrm{III}}\left(x ; \frac{1}{4}\right), 1 / \tilde{\phi}_{1}^{\mathrm{III}}\left(x ; \frac{1}{4}\right)\right)=\infty .
\end{aligned}
$$

A cubic zero of the seed solution in (A) and (B) is realised by a nice magic of the Wronskian

$$
\begin{gathered}
\mathrm{W}\left[e^{x^{2} / 2} x^{1 / 4}\left(3+4 x^{2}\right), e^{x^{2} / 2} x^{3 / 4}\left(3+4 x^{2}\right)\left(15+4 x^{2}\right)\right](x) \\
=\left(e^{x^{2} / 2} x^{1 / 4}\left(3+4 x^{2}\right)\right)^{2} \mathrm{~W}\left[1, x^{1 / 2}\left(15+4 x^{2}\right)\right](x) .
\end{gathered}
$$

The Wronskian on the r.h.s. provides another factor $3+4 x^{2}$. The fact that $1 / \tilde{\phi}_{1}^{\mathrm{I}}\left(x ; \frac{1}{4}\right)$ and $1 / \tilde{\phi}_{1}^{\mathrm{III}}\left(x ; \frac{3}{4}\right)$ are square integrable (3.13) means that the mechanism of multi-index polynomials developed in [19] does not apply.

For $(\mathrm{C})$ and $(\mathrm{D})$, the functions are

$$
\begin{array}{ll}
\tilde{\phi}_{1}^{\mathrm{II}}\left(x ; \frac{9}{4}\right)=-\frac{1}{4} e^{-x^{2} / 2} x^{-5 / 4}\left(3+4 x^{2}\right), & \tilde{\phi}_{2}^{\mathrm{II}}\left(x ; \frac{9}{4}\right)=\frac{1}{32} e^{-x^{2} / 2} x^{-5 / 4}\left(-3+4 x^{2}\right)\left(1+4 x^{2}\right), \\
\tilde{\phi}_{1}^{\mathrm{III}}\left(x ; \frac{9}{4}\right)=\frac{1}{4} e^{x^{2} / 2} x^{-5 / 4}\left(-3+4 x^{2}\right), & \tilde{\phi}_{2}^{\mathrm{III}}\left(x ; \frac{9}{4}\right)=\frac{1}{32} e^{x^{2} / 2} x^{-5 / 4}\left(3+4 x^{2}\right)\left(-1+4 x^{2}\right), \\
\left(\tilde{\phi}_{1}^{\mathrm{II}}\left(x ; \frac{9}{4}\right), \tilde{\phi}_{1}^{\mathrm{II}}\left(x ; \frac{9}{4}\right)\right)=\infty, \quad\left(1 / \tilde{\phi}_{1}^{\mathrm{II}}\left(x ; \frac{9}{4}\right), 1 / \tilde{\phi}_{1}^{\mathrm{II}}\left(x ; \frac{9}{4}\right)\right)=\infty .
\end{array}
$$

Again the cubic zeros of these cases are realised by a nice magic of the Wronskian. As expected, $\tilde{\phi}_{2}^{\mathrm{II}}\left(x ; \frac{9}{4}\right), \tilde{\phi}_{1}^{\mathrm{III}}\left(x ; \frac{9}{4}\right)$ and $\tilde{\phi}_{2}^{\mathrm{III}}\left(x ; \frac{9}{4}\right)$ change sign in $(0, \infty)$, which is a deviation from the mechanism of multi-index polynomials developed in [19].

Now it is clear that the solutions $\phi_{n}^{(2)}(x)(3.6)$ have a $\rho=-2$ apparent singularity, that is,

$$
\phi_{n}^{(2)}(x) \propto \frac{1}{\left(3+4 x^{2}\right)^{2}}, \quad(\mathrm{~A}), \quad(\mathrm{B}), \quad(\mathrm{C}), \quad \phi_{n}^{(2)}(x) \propto \frac{1}{\left(-3+4 x^{2}\right)^{2}}, \quad(\mathrm{D}) .
$$

Among the six terms of the numerator Wronskian $\mathrm{W}\left[\varphi_{1}, \varphi_{2}, \phi_{n}\right](x)$ of (3.6), the four terms containing $\phi_{n}^{\prime}(x)$ or $\phi_{n}^{\prime \prime}(x)$ contain at least one term $\varphi_{1}(x)$ or $\varphi_{2}(x)$ without a derivative. Thus they contain at least one factor of $3+4 x^{2}$ in (A), (B), (C) and $-3+4 x^{2}$ in (D). The remaining two terms which contain $\phi_{n}(x)$ are proportional to

$$
\varphi_{1}^{\prime}(x) \varphi_{2}^{\prime \prime}(x)-\varphi_{1}^{\prime \prime}(x) \varphi_{2}^{\prime}(x) .
$$

Since $\varphi_{j}(x)$ is a solution of the original Schrödinger equation (2.1), they satisfy

$$
\varphi_{j}^{\prime \prime}(x)=\left(U(x)-\tilde{\mathcal{E}}_{j}\right) \varphi_{j}(x), \quad j=1,2 .
$$

Thus the above terms also contain one factor of $\varphi_{1}(x)$ or $\varphi_{2}(x)$, that is, $3+4 x^{2}$ in (A), (B), (C) and $-3+4 x^{2}$ in (D). Thus one factor of the cubic zero of the seed function $\mathrm{W}\left[\varphi_{1}, \varphi_{2}\right](x)$ is cancelled and we arrive at (3.14). 
The relationship between the generic cases $(\mathrm{E})$ and $(\mathrm{F})$ has similarities with that between the non-generic cases $(\mathrm{C})$ and $(\mathrm{D})$. The functions in $(\mathrm{E})$ are

$$
\tilde{\phi}_{2}^{\mathrm{I}}\left(x ; \frac{15}{2}\right)=\frac{1}{2} e^{x^{2} / 2} x^{15 / 2}\left(6+x^{2}\right)\left(12+x^{2}\right), \quad \tilde{\phi}_{1}^{\mathrm{II}}\left(x ; \frac{15}{2}\right)=-e^{-x^{2} / 2} x^{-13 / 2}\left(6+x^{2}\right) .
$$

The functions in $(\mathrm{F})$ are

$$
\tilde{\phi}_{1}^{\mathrm{I}}\left(x ;-\frac{13}{2}\right)=e^{x^{2} / 2} x^{-13 / 2}\left(-6+x^{2}\right), \quad \tilde{\phi}_{1}^{\mathrm{II}}\left(x ;-\frac{13}{2}\right)=\frac{1}{2} e^{-x^{2} / 2} x^{15 / 2}\left(-6+x^{2}\right)\left(-12+x^{2}\right) .
$$

Thus it is also clear that the solutions $\phi_{n}^{(2)}(x)(3.6)$ have a $\rho=-2$ apparent singularity, that is,

$$
\phi_{n}^{(2)}(x) \propto \frac{1}{\left(6+x^{2}\right)^{2}}, \quad(\mathrm{E}), \quad \phi_{n}^{(2)}(x) \propto \frac{1}{\left(x^{2}-6\right)^{2}}, \quad(\mathrm{~F}) .
$$

\subsection{Global solutions with a $\rho=-2$ apparent singularity}

Here we present the infinitely many global solutions with a $\rho=-2$ apparent singularity in explicit forms. They are simply obtained by evaluating $\phi_{n}^{(2)}(x)(3.6)$ in each case $(\mathrm{A})-(\mathrm{F})$, to be denoted by $\phi_{n}^{A}(x), \phi_{n}^{B}(x), \ldots, \phi_{n}^{F}(x)$, respectively. Most formulas look simpler as functions of $\eta \equiv \eta(x)=x^{2}$, since the eigenpolynomials of the radial oscillator potential are the Laguerre polynomials in $\eta,(3.2)$.

\subsubsection{Global solutions (A)}

The global solutions are

$$
\begin{aligned}
& \mathcal{H}^{A} \phi_{n}^{A}(x)=\mathcal{E}_{n} \phi_{n}^{A}(x), \quad \mathcal{H}^{A} \stackrel{\text { def }}{=}-\frac{d^{2}}{d x^{2}}+U_{A}(x), \quad \mathcal{E}_{n}=4 n, \\
& \phi_{n}^{A}(x)=\frac{e^{-\eta / 2} \eta^{3 / 8}}{(3+4 \eta)^{2}} \mathcal{L}_{n}^{A}(\eta), \quad n=0,1, \ldots,
\end{aligned}
$$

in which $\mathcal{L}_{n}^{A}(\eta)(n \geq 0)$ is a degree $n+3$ polynomial in $\eta$ defined by

$$
\begin{aligned}
\mathcal{L}_{n}^{A}(\eta) \stackrel{\text { def }}{=} & \left(-117+156 \eta+208 \eta^{2}+64 \eta^{3}\right) L_{n}^{(1 / 4)}(\eta) \\
& -4 n(3+4 \eta)^{2} L_{n}^{(1 / 4)}(\eta)-4 \eta(3+4 \eta)(15+4 \eta) \partial_{\eta} L_{n}^{(1 / 4)}(\eta) .
\end{aligned}
$$

In evaluating the Wronskian $\mathrm{W}\left[\tilde{\phi}_{1}^{\mathrm{III}}\left(x ; \frac{3}{4}\right), \tilde{\phi}_{2}^{\mathrm{I}}\left(x ; \frac{3}{4}\right), \phi_{n}\left(x ; \frac{3}{4}\right)\right](x)$, the second derivative of the Laguerre polynomial $L_{n}^{(1 / 4)}(\eta)$ appears. It is replaced by $L_{n}^{(1 / 4)}(\eta)$ and $\partial_{\eta} L_{n}^{(1 / 4)}(\eta)$ by using the equation for the Laguerre polynomial

$$
x \partial_{x}^{2} L_{n}^{(\alpha)}(x)+(\alpha+1-x) \partial_{x} L_{n}^{(\alpha)}(x)+n L_{n}^{(\alpha)}(x)=0 .
$$

There is another member of this family corresponding to the eigenvalue $\mathcal{E}=-8$, because of the fact that the Darboux transformation in terms of $\tilde{\phi}_{1}^{\mathrm{III}}\left(x ; \frac{3}{4}\right)$ is not one-to-one, $\left(1 / \tilde{\phi}_{1}^{\mathrm{III}}\left(x ; \frac{3}{4}\right)\right.$, $\left.1 / \tilde{\phi}_{1}^{\mathrm{III}}\left(x ; \frac{3}{4}\right)\right)<\infty,(3.13)$, see [19]. Let us denote it tentatively by $\mathcal{L}_{-2}^{A}(\eta)$

$$
\mathcal{L}_{-2}^{A}(\eta) \stackrel{\text { def }}{=} 15+4 \eta
$$

whose normalisation is irrelevant. Note that $n=-1$ is missing. Some lower members are

$$
\begin{aligned}
& \mathcal{L}_{0}^{A}(\eta)=-117+156 \eta+208 \eta^{2}+64 \eta^{3}, \quad \mathcal{L}_{1}^{A}(\eta)=-\frac{765}{4}+408 \eta+408 \eta^{2}-64 \eta^{4} \\
& \mathcal{L}_{2}^{A}(\eta)=-\frac{8505}{32}+\frac{6237}{8} \eta+567 \eta^{2}-252 \eta^{3}-168 \eta^{4}+32 \eta^{5}
\end{aligned}
$$


They are also discussed in $\S 6.2 .5$ of [9]. They satisfy the orthogonality relation

$$
\begin{aligned}
& \int_{0}^{\infty} \frac{e^{-\eta} \eta^{\frac{1}{4}}}{(3+4 \eta)^{4}} \mathcal{L}_{n}^{A}(\eta) \mathcal{L}_{m}^{A}(\eta) d \eta=h_{n}^{A} \delta_{n m}, \quad n, m=-2,0,1, \ldots, \\
& h_{n}^{A} \stackrel{\text { def }}{=} \frac{4(n+2)(4 n+13) \Gamma\left(n+\frac{5}{4}\right)}{n !}, \quad n \neq-2,
\end{aligned}
$$

which is obtained by rewriting $(2.4)$ with $\tilde{\mathcal{E}}_{1}^{\mathrm{III}}\left(\frac{3}{4}\right)=-8$ and $\tilde{\mathcal{E}}_{2}^{\mathrm{I}}\left(\frac{3}{4}\right)=-13$.

By rewriting the Schrödinger equation for $\phi_{n}^{A}(x)$ (3.15) in terms of

$$
\phi_{n}^{A}(x)=e^{-\eta / 2} \eta^{3 / 8} y(\eta),
$$

we obtain a second-order differential equation with regular a singularity at $\eta=0$ and $\eta=-\frac{3}{4}$ and an irregular singularity at $x=\infty$

$$
\eta y^{\prime \prime}+\left(\frac{5}{4}-\eta\right) y^{\prime}+\frac{45-24 \eta+16 \eta^{2}}{(3+4 \eta)^{2}} y+n y=0, \quad n=-2,0,1, \ldots
$$

For each $n$ we have a global solution with a $\rho=-2$ apparent singularity

$$
y_{n}(\eta)=\frac{\mathcal{L}_{n}^{A}(\eta)}{(3+4 \eta)^{2}}, \quad n=-2,0,1, \ldots
$$

The differential equations for the polynomials $\left\{\mathcal{L}_{n}^{A}(\eta)\right\}$ read

$$
\begin{aligned}
4 \eta(3+4 \eta) \mathcal{L}_{n}^{A}(\eta)^{\prime \prime}-(4 \eta-1)(15+4 \eta) \mathcal{L}_{n}^{A}(\eta)^{\prime} & \\
& +4(5+12 \eta+n(3+4 \eta)) \mathcal{L}_{n}^{A}(\eta)=0, \quad n=-2,0,1, \ldots
\end{aligned}
$$

The groundstate wavefunction $\phi_{-2}^{A}(x)(3.16)$ with $\mathcal{L}_{-2}^{A}$ (3.17) and the energy $\mathcal{E}=-8$ is related to the potential $U_{A}(x)$ (3.7) through the prepotential $w_{A}(x)$ (Riccati form of Schrödinger equation)

$$
e^{w_{A}(x)} \stackrel{\text { def }}{=} \frac{e^{-x^{2} / 2} x^{3 / 4}\left(15+4 x^{2}\right)}{\left(3+4 x^{2}\right)^{2}} \Rightarrow\left(\frac{d w_{A}(x)}{d x}\right)^{2}+\frac{d^{2} w_{A}(x)}{d x^{2}}-8=U_{A}(x) .
$$

In other words, the Hamiltonian $\mathcal{H}^{A}(3.15)$ is written in a factorised form

$$
\mathcal{H}^{A}=\mathcal{A}^{A \dagger} \mathcal{A}^{A}-8, \quad \mathcal{A}^{A} \stackrel{\text { def }}{=} \frac{d}{d x}-\frac{d w_{A}(x)}{d x}, \quad \mathcal{A}^{A \dagger}=-\frac{d}{d x}-\frac{d w_{A}(x)}{d x} .
$$

It is easy to verify that the standard Darboux-Crum transformation [3] of deleting the groundstate applied to (3.19) will lead to the system of one-indexed (or the exceptional) polynomial system generated in terms of the virtual state solution $\tilde{\phi}_{2}^{\mathrm{I}}\left(x ; \frac{3}{4}\right)$

$$
\begin{aligned}
& \mathcal{H}^{A 1} \stackrel{\text { def }}{=} \mathcal{A}^{A} \mathcal{A}^{A \dagger}-8=-\frac{d^{2}}{d x^{2}}+\left(\frac{d w_{A}(x)}{d x}\right)^{2}-\frac{d^{2} w_{A}(x)}{d x^{2}}-8=-\frac{d^{2}}{d x^{2}}+U_{A 1}(x), \\
& U_{A 1}(x)=x^{2}+\frac{21}{16 x^{2}}+\frac{16}{3+4 x^{2}}-\frac{96}{\left(3+4 x^{2}\right)^{2}}+\frac{16}{15+4 x^{2}}-\frac{480}{\left(15+4 x^{2}\right)^{2}}-\frac{9}{2} \\
& =U\left(x ; \frac{3}{4}\right)-2 \frac{d^{2}}{d x^{2}} \log \tilde{\phi}_{2}^{\mathrm{I}}\left(x ; \frac{3}{4}\right) .
\end{aligned}
$$

This shows clearly that the non-generic two-indexed polynomial system (A) generated by a nonvirtual state solution $\tilde{\phi}_{1}^{\mathrm{III}}\left(x ; \frac{3}{4}\right)$ and a virtual state solution $\tilde{\phi}_{2}^{\mathrm{I}}\left(x ; \frac{3}{4}\right)$ is not shape-invariant [7], in contrast with the generic multi-indexed polynomial systems [19]. The standard Darboux-Crum transformation removes the groundstate "created" by the non-virtual state solution $\tilde{\phi}_{1}^{\mathrm{III}}\left(x ; \frac{3}{4}\right)$ and the generic one-indexed polynomial system generated by the virtual state solution $\tilde{\phi}_{2}^{\mathrm{I}}\left(x ; \frac{3}{4}\right)$ is obtained. 


\subsubsection{Global solutions (B)}

The global solutions are

$$
\begin{aligned}
& \mathcal{H}^{B} \phi_{n}^{B}(x)=\mathcal{E}_{n} \phi_{n}^{B}(x), \quad \mathcal{H}^{B} \stackrel{\text { def }}{=}-\frac{d^{2}}{d x^{2}}+U_{B}(x), \quad \mathcal{E}_{n}=4 n, \\
& \phi_{n}^{B}(x)=\frac{e^{-\eta / 2} \eta^{1 / 8}}{(3+4 \eta)^{2}} \mathcal{L}_{n}^{B}(\eta), \quad n=0,1, \ldots,
\end{aligned}
$$

in which $\mathcal{L}_{n}^{B}(\eta)(n \geq 0)$ is a degree $n+3$ polynomial in $\eta$ defined by

$$
\begin{aligned}
\mathcal{L}_{n}^{B}(\eta) \stackrel{\text { def }}{=} & \left(-63+252 \eta+240 \eta^{2}+64 \eta^{3}\right) L_{n}^{(-1 / 4)}(\eta) \\
& -4 n(3+4 \eta)^{2} L_{n}^{(-1 / 4)}(\eta)-4 \eta(3+4 \eta)(15+4 \eta) \partial_{\eta} L_{n}^{(-1 / 4)}(\eta) .
\end{aligned}
$$

There is another member of this family corresponding to the eigenvalue $\mathcal{E}=-12$, because of the fact that the Darboux transformation in terms of $\tilde{\phi}_{2}^{\mathrm{III}}\left(x ; \frac{1}{4}\right)$ is not one to one, $\left(1 / \tilde{\phi}_{2}^{\mathrm{III}}\left(x ; \frac{1}{4}\right)\right.$, $\left.1 / \tilde{\phi}_{2}^{\mathrm{III}}\left(x ; \frac{1}{4}\right)\right)<\infty,(3.13)$, see [19]. Let us denote it tentatively by $\mathcal{L}_{-3}^{B}(\eta)$

$$
\mathcal{L}_{-3}^{B}(\eta) \stackrel{\text { def }}{=} 1 .
$$

Its normalisation is irrelevant. Note that $n=-2,-1$ are missing. Some lower-degree members are

$$
\begin{aligned}
& \mathcal{L}_{0}^{B}(\eta)=-63+252 \eta+240 \eta^{2}+64 \eta^{3}, \quad \mathcal{L}_{1}^{B}(\eta)=-\frac{297}{4}+396 \eta+264 \eta^{2}-64 \eta^{3}-64 \eta^{4} \\
& \mathcal{L}_{2}^{B}(\eta)=-\frac{2835}{32}+\frac{4725}{8} \eta+225 \eta^{2}-300 \eta^{3}-120 \eta^{4}+32 \eta^{5}
\end{aligned}
$$

They are also discussed in $\S 6.2 .6$ of [9]. They satisfy the orthogonality relation

$$
\begin{aligned}
& \int_{0}^{\infty} \frac{e^{-\eta} \eta^{-1 / 4}}{(3+4 \eta)^{4}} \mathcal{L}_{n}^{B}(\eta) \mathcal{L}_{m}^{B}(\eta) d \eta=h_{n}^{B} \delta_{n m}, \quad n, m=-3,0,1, \ldots, \\
& h_{n}^{B} \stackrel{\text { def }}{=} \frac{4(n+3)(4 n+7) \Gamma\left(n+\frac{3}{4}\right)}{n !}, \quad n \neq-3,
\end{aligned}
$$

which is obtained by rewriting $(2.4)$ with $\tilde{\mathcal{E}}_{2}^{\mathrm{III}}\left(\frac{3}{4}\right)=-12$ and $\tilde{\mathcal{E}}_{1}^{\mathrm{I}}\left(\frac{3}{4}\right)=-7$.

By rewriting the Schrödinger equation for $\phi_{n}^{B}(x)(3.20)$ in terms of

$$
\phi_{n}^{B}(x)=e^{-\eta / 2} \eta^{1 / 8} y(\eta),
$$

we obtain a second-order differential equation with a regular singularity at $\eta=0$ and $\eta=-\frac{3}{4}$ and an irregular singularity at $x=\infty$

$$
\eta y^{\prime \prime}+\left(\frac{3}{4}-\eta\right) y^{\prime}+\frac{45-24 \eta+16 \eta^{2}}{(3+4 \eta)^{2}} y+n y=0, \quad n=-3,0,1, \ldots
$$

For each $n$ we have a global solution with a $\rho=-2$ apparent singularity

$$
y_{n}(\eta)=\frac{\mathcal{L}_{n}^{B}(\eta)}{(3+4 \eta)^{2}}, \quad n=-3,0,1, \ldots
$$

The differential equations for the polynomials $\left\{\mathcal{L}_{n}^{B}(\eta)\right\}$ read

$$
\begin{aligned}
4 \eta(3+4 \eta) \mathcal{L}_{n}^{B}(\eta)^{\prime \prime}-\left(-9+64 \eta+16 \eta^{2}\right) \mathcal{L}_{n}^{B}(\eta)^{\prime} \\
\quad+4(3+n)(3+4 \eta) \mathcal{L}_{n}^{B}(\eta)=0, \quad n=-3,0,1, \ldots
\end{aligned}
$$


The groundstate wavefunction $\phi_{-3}^{B}(x)$ (3.21) with $\mathcal{L}_{-3}^{B}$ (3.22) and the energy $\mathcal{E}=-12$ is related to the potential $U_{B}(x)$ (3.8) through the prepotential $w_{B}(x)$ (Riccati form of Schrödinger equation)

$$
e^{w_{B}(x)} \stackrel{\text { def }}{=} \frac{e^{-x^{2} / 2} x^{1 / 4}}{\left(3+4 x^{2}\right)^{2}} \Rightarrow\left(\frac{d w_{B}(x)}{d x}\right)^{2}+\frac{d^{2} w_{B}(x)}{d x^{2}}-12=U_{B}(x) .
$$

In other words, the Hamiltonian $\mathcal{H}^{B}$ (3.20) is written in a factorised form

$$
\mathcal{H}^{B}=\mathcal{A}^{B \dagger} \mathcal{A}^{B}-12, \quad \mathcal{A}^{B} \stackrel{\text { def }}{=} \frac{d}{d x}-\frac{d w_{B}(x)}{d x}, \quad \mathcal{A}^{B \dagger}=-\frac{d}{d x}-\frac{d w_{B}(x)}{d x} .
$$

It is easy to verify that the standard Darboux-Crum transformation [3] of deleting the groundstate will lead to the system of one-indexed (or the exceptional) polynomial system generated in terms of the virtual state solution $\tilde{\phi}_{1}^{\mathrm{I}}\left(x ; \frac{1}{4}\right)$

$$
\begin{aligned}
& \mathcal{H}^{B 1} \stackrel{\text { def }}{=} \mathcal{A}^{B} \mathcal{A}^{B \dagger}-12=-\frac{d^{2}}{d x^{2}}+\left(\frac{d w_{B}(x)}{d x}\right)^{2}-\frac{d^{2} w_{B}(x)}{d x^{2}}-12=-\frac{d^{2}}{d x^{2}}+U_{B 1}(x), \\
& U_{B 1}(x)=x^{2}+\frac{5}{16 x^{2}}+\frac{16}{3+4 x^{2}}-\frac{96}{\left(3+4 x^{2}\right)^{2}}-\frac{7}{2}=U\left(x ; \frac{1}{4}\right)-2 \frac{d^{2}}{d x^{2}} \log \tilde{\phi}_{1}^{\mathrm{I}}\left(x ; \frac{1}{4}\right) .
\end{aligned}
$$

\subsubsection{Global solutions (D)}

The global solutions are

$$
\begin{aligned}
& \mathcal{H}^{D} \phi_{n}^{D}(x)=\mathcal{E}_{n} \phi_{n}^{D}(x), \quad \mathcal{H}^{D} \stackrel{\text { def }}{=}-\frac{d^{2}}{d x^{2}}+U_{D}(x), \quad \mathcal{E}_{n}=4 n, \\
& \phi_{n}^{D}(x)=\frac{e^{-\eta / 2} \eta^{1 / 8}}{4(-3+4 \eta)^{2}} \mathcal{L}_{n}^{D}(\eta), \quad n=0,1, \ldots,
\end{aligned}
$$

in which $\mathcal{L}_{n}^{D}(\eta)(n \geq 0)$ is a degree $n+3$ polynomial in $\eta$ defined by

$$
\begin{aligned}
\mathcal{L}_{n}^{D}(\eta) \stackrel{\text { def }}{=} & (3+4 \eta)\left(63+16 \eta^{2}\right) L_{n}^{(7 / 4)}(\eta) \\
& -16 n \eta(-3+4 \eta)^{2} L_{n}^{(7 / 4)}(\eta)-36 \eta(-3+4 \eta)(1+4 \eta) \partial_{\eta} L_{n}^{(7 / 4)}(\eta) .
\end{aligned}
$$

There is another member of this family belonging to the energy $\mathcal{E}=-8$, to be denoted tentatively by $\mathcal{L}_{-2}^{D}(\eta)$

$$
\mathcal{L}_{-2}^{D}(\eta) \stackrel{\text { def }}{=} 1+4 \eta
$$

Some lower members are

$$
\begin{aligned}
& \mathcal{L}_{0}^{D}(\eta)=(3+4 \eta)\left(63+16 \eta^{2}\right), \quad \mathcal{L}_{1}^{D}(\eta)=\frac{2079}{4}+792 \eta^{2}-384 \eta^{3}+192 \eta^{4}, \\
& \mathcal{L}_{2}^{D}(\eta)=\frac{31185}{32}-\frac{10395}{8} \eta+3465 \eta^{2}-2940 \eta^{3}+1512 \eta^{4}-224 \eta^{5}
\end{aligned}
$$

Note that $n=-1$ is missing. The lowest member $\mathcal{L}_{-2}^{D}(3.25)$ with the energy $\mathcal{E}=-8$ is related to the potential $U_{D}(x)$ (3.10) through the prepotential (Riccati form of Schrödinger equation)

$$
e^{w_{D}(x)} \stackrel{\text { def }}{=} \frac{e^{-x^{2} / 2} x^{1 / 4}\left(1+4 x^{2}\right)}{\left(-3+4 x^{2}\right)^{2}} \Rightarrow\left(\frac{d w_{D}(x)}{d x}\right)^{2}+\frac{d^{2} w_{D}(x)}{d x^{2}}-8=U_{D}(x) .
$$


By rewriting the Schrödinger equation for $\phi_{n}^{D}(x)$ (3.24) in terms of

$$
\phi_{n}^{D}(x)=e^{-\eta / 2} \eta^{1 / 8} y(\eta),
$$

we obtain a second-order differential equation with a regular singularity at $\eta=0$ and $\eta=+\frac{3}{4}$ and an irregular singularity at $x=\infty$

$$
\eta y^{\prime \prime}+\left(\frac{3}{4}-\eta\right) y^{\prime}-\frac{27+72 \eta-16 \eta^{2}}{(-3+4 \eta)^{2}} y+n y=0, \quad n=-2,0,1, \ldots
$$

For each $n$ we have a global solution with a $\rho=-2$ apparent singularity:

$$
y_{n}(\eta)=\frac{\mathcal{L}_{n}^{D}(\eta)}{(-3+4 \eta)^{2}}, \quad n=-2,0,1, \ldots
$$

The differential equations for the polynomials $\left\{\mathcal{L}_{n}^{D}(\eta)\right\}$ read

$$
\begin{aligned}
4 \eta(-3 & +4 \eta) \mathcal{L}_{n}^{D}(\eta)^{\prime \prime}-(1+4 \eta)(9+4 \eta) \mathcal{L}_{n}^{D}(\eta)^{\prime} \\
& +4(3+12 \eta+n(-3+4 \eta)) \mathcal{L}_{n}^{D}(\eta)=0, \quad n=-2,0,1, \ldots
\end{aligned}
$$

\subsubsection{Global solutions (E)}

The global solutions are

$$
\begin{aligned}
& \mathcal{H}^{E} \phi_{n}^{E}(x)=\mathcal{E}_{n} \phi_{n}^{E}(x), \quad \mathcal{H}^{E} \stackrel{\text { def }}{=}-\frac{d^{2}}{d x^{2}}+U_{E}(x), \quad \mathcal{E}_{n}=4 n, \\
& \phi_{n}^{E}(x)=\frac{e^{-\eta / 2} \eta^{\frac{15}{4}}}{(6+\eta)^{2}(14+\eta)} \mathcal{L}_{n}^{E}(\eta), \quad n=0,1, \ldots,
\end{aligned}
$$

in which $\mathcal{L}_{n}^{E}(\eta)$ is a degree $n+3$ polynomial in $\eta$ defined by

$$
\begin{aligned}
\mathcal{L}_{n}^{E}(\eta) \stackrel{\text { def }}{=} & -24\left(840+280 \eta+30 \eta^{2}+\eta^{3}\right) L_{n}^{(7)}(\eta) \\
& -4 n(6+\eta)^{2}(14+\eta) L_{n}^{(7)}(\eta)-16 \eta(6+\eta)(12+\eta) \partial_{\eta} L_{n}^{(7)}(\eta) .
\end{aligned}
$$

This belongs to the generic case and the Darboux transformation is one-to-one. The groundstate corresponds to $\mathcal{L}_{0}^{E}$. Some lower members are

$$
\begin{aligned}
& \mathcal{L}_{0}^{E}(\eta)=-24\left(840+280 \eta+30 \eta^{2}+\eta^{3}\right), \\
& \mathcal{L}_{1}^{E}(\eta)=28\left(-6336-1320 \eta+44 \eta^{2}+22 \eta^{3}+\eta^{4}\right), \\
& \mathcal{L}_{2}^{E}(\eta)=-16\left(54432+4536 \eta-1944 \eta^{2}-180 \eta^{3}+12 \eta^{4}+\eta^{5}\right) .
\end{aligned}
$$

They satisfy the orthogonality relation

$$
\begin{aligned}
& \int_{0}^{\infty} \frac{e^{-\eta} \eta^{7}}{(6+\eta)^{4}(14+\eta)^{2}} \mathcal{L}_{n}^{E}(\eta) \mathcal{L}_{m}^{E}(\eta) d \eta=h_{n}^{E} \delta_{n m}, \quad n, m=0,1, \ldots, \\
& h_{n}^{E} \stackrel{\text { def }}{=} 16(n+10)(n+6)(n+1)_{7},
\end{aligned}
$$

which is obtained by rewriting $(2.4)$ with $\tilde{\mathcal{E}}_{2}^{\mathrm{I}}\left(\frac{15}{2}\right)=-40$ and $\tilde{\mathcal{E}}_{1}^{\mathrm{II}}\left(\frac{15}{2}\right)=-24$. Here $(a)_{n} \stackrel{\text { def }}{=}$ $\prod_{k=1}^{n}(a+k-1)$ is the shifted factorial (the Pochhammer symbol).

By rewriting the Schrödinger equation for $\phi_{n}^{E}(x)(3.27)$ in terms of

$$
\phi_{n}^{E}(x)=e^{-\eta / 2} \eta^{15 / 4} y(\eta),
$$


we obtain a second-order differential equation with a regular singularity at $\eta=0$ and $\eta=$ $-6,-14$ and an irregular singularity at $x=\infty$

$$
\eta y^{\prime \prime}+(8-\eta) y^{\prime}+4 \frac{1008+12 \eta-16 \eta^{2}-\eta^{3}}{(6+\eta)^{2}(14+\eta)^{2}} y+n y=0, \quad n=0,1, \ldots
$$

For each $n$ we have a global solution with a $\rho=-2$ and a $\rho=-1$ apparent singularities:

$$
y_{n}(\eta)=\frac{\mathcal{L}_{n}^{E}(\eta)}{(6+\eta)^{2}(14+\eta)}, \quad n=0,1, \ldots
$$

The differential equations for the polynomials $\left\{\mathcal{L}_{n}^{E}(\eta)\right\}$ read

$$
\begin{aligned}
\eta(6+\eta)(14+\eta) \mathcal{L}_{n}^{E}(\eta)^{\prime \prime}-\left(-672-8 \eta+18 \eta^{2}+\eta^{3}\right) \mathcal{L}_{n}^{E}(\eta)^{\prime} & \\
& +\left(-224+18 \eta+3 \eta^{2}+n(6+\eta)(14+\eta)\right) \mathcal{L}_{n}^{E}(\eta)=0, \quad n=0,1, \ldots
\end{aligned}
$$

The groundstate wavefunction $\phi_{0}^{E}(x)(3.28)$ with $\mathcal{L}_{0}^{E}(3.29)$ is related to the potential $U_{E}(x)$ (3.11) through the prepotential $w_{E}(x)$ :

$$
e^{w_{E}(x)} \stackrel{\text { def }}{=} \frac{e^{-x^{2} / 2} x^{15 / 2}\left(840+280 x^{2}+30 x^{4}+x^{6}\right)}{\left(6+x^{2}\right)^{2}\left(14+x^{2}\right)} \Rightarrow\left(\frac{d w_{E}(x)}{d x}\right)^{2}+\frac{d^{2} w_{E}(x)}{d x^{2}}=U_{E}(x) .
$$

By construction, the system is shape-invariant

$$
\begin{gathered}
\left(\frac{d w_{E}(x)}{d x}\right)^{2}-\frac{d^{2} w_{E}(x)}{d x^{2}}=U\left(x ; \frac{17}{2}\right)-2 \frac{d^{2}}{d x^{2}} \log \mathrm{W}\left[\tilde{\phi}_{2}^{\mathrm{I}}\left(x ; \frac{17}{2}\right), \tilde{\phi}_{1}^{\mathrm{II}}\left(x ; \frac{17}{2}\right)\right](x)+4 \\
=x^{2}+\frac{255}{4 x^{2}}-14-\frac{48}{\left(6+x^{2}\right)^{2}}+\frac{4}{6+x^{2}} \\
\quad-\frac{640\left(630+175 x^{2}+12 x^{4}\right)}{\left(840+280 x^{2}+30 x^{4}+x^{6}\right)^{2}}+\frac{4\left(-160+3 x^{2}\right)}{\left(840+280 x^{2}+30 x^{4}+x^{6}\right)} .
\end{gathered}
$$

As shown clearly above, the singularity at $x^{2}=-6$ of the new potential corresponds to the characteristic exponents $\rho=-1,2$.

\subsubsection{Global solutions (F)}

The global solutions are singular in $(0, \infty)$

$$
\begin{aligned}
& \mathcal{H}^{F} \phi_{n}^{F}(x)=\mathcal{E}_{n} \phi_{n}^{F}(x), \quad \mathcal{H}^{F} \stackrel{\text { def }}{=}-\frac{d^{2}}{d x^{2}}+U_{F}(x), \quad \mathcal{E}_{n}=4 n, \\
& \phi_{n}^{F}(x)=\frac{e^{-\eta / 2} \eta^{-13 / 4}}{(-6+\eta)^{2}(-14+\eta)} \mathcal{L}_{n}^{F}(\eta), \quad n=0,1, \ldots,
\end{aligned}
$$

in which $\mathcal{L}_{n}^{F}(\eta)$ is a degree $n+3$ polynomial in $\eta$ defined by

$$
\begin{aligned}
\mathcal{L}_{n}^{F}(\eta) \stackrel{\text { def }}{=} & -4\left(-9\left(-280+140 \eta-22 \eta^{2}+\eta^{3}\right)+n(-14+\eta)(-6+\eta)\right) L_{n}^{(-7)}(\eta) \\
& -16 \eta(-6+\eta)(-12+\eta) \partial_{\eta} L_{n}^{(-7)}(\eta) .
\end{aligned}
$$

They are not square integrable and thus they are not eigenfunctions. Some lower members are

$$
\begin{aligned}
& \mathcal{L}_{0}^{F}(\eta)=36\left(-280+140 \eta-22 \eta^{2}+\eta^{3}\right), \\
& \mathcal{L}_{1}^{F}(\eta)=-32\left(-1512+504 \eta+12 \eta^{2}-16 \eta^{3}+\eta^{4}\right), \\
& \mathcal{L}_{2}^{F}(\eta)=14\left(-6480+1080 \eta+396 \eta^{2}-42 \eta^{3}-12 \eta^{4}+\eta^{5}\right) .
\end{aligned}
$$


By rewriting the Schrödinger equation for $\phi_{n}^{F}(x)(3.31)$ in terms of

$$
\phi_{n}^{F}(x)=e^{-\eta / 2} \eta^{-13 / 4} y(\eta)
$$

we obtain a second-order differential equation with a regular singularity at $\eta=0$ and $\eta=6,14$ and an irregular singularity at $x=\infty$

$$
\eta y^{\prime \prime}-(6+\eta) y^{\prime}-4 \frac{1008-12 \eta-16 \eta^{2}+\eta^{3}}{(-6+\eta)^{2}(-14+\eta)^{2}} y+n y=0, \quad n=0,1, \ldots
$$

For each $n$ we have a singular global solution with a $\rho=-2$ and a $\rho=-1$ apparent singularities

$$
y_{n}(\eta)=\frac{\mathcal{L}_{n}^{F}(\eta)}{(-6+\eta)^{2}(-14+\eta)}, \quad n=0,1, \ldots
$$

The differential equations for the polynomials $\left\{\mathcal{L}_{n}^{F}(\eta)\right\}$ read

$$
\begin{aligned}
\eta(-6 & +\eta)(-14+\eta) \mathcal{L}_{n}^{F}(\eta)^{\prime \prime}-\left(504-104 \eta-8 \eta^{2}+\eta^{3}\right) \mathcal{L}_{n}^{F}(\eta)^{\prime} \\
& +\left(-252-8 \eta+3 \eta^{2}+n(-6+\eta)(-14+\eta)\right) \mathcal{L}_{n}^{F}(\eta)=0, \quad n=0,1, \ldots
\end{aligned}
$$

The wavefunction $\phi_{0}^{F}(x)(3.32)$ with $\mathcal{L}_{0}^{F}$ (3.33) is related to the potential $U_{F}(x)$ (3.12) through the prepotential $w_{F}(x)$

$$
\begin{gathered}
e^{w_{F}(x) \stackrel{\text { def }}{=}} \frac{e^{-x^{2} / 2} x^{-13 / 2}\left(-280+140 x^{2}-22 x^{4}+x^{6}\right)}{\left(-6+x^{2}\right)^{2}\left(-14+x^{2}\right)} \\
\Rightarrow\left(\frac{d w_{F}(x)}{d x}\right)^{2}+\frac{d^{2} w_{F}(x)}{d x^{2}}=U_{F}(x) .
\end{gathered}
$$

By construction, the system is shape-invariant. The equations and the wavefunctions of (E) and $(\mathrm{F})$ systems have very similar forms.

Here we provide a summary table of $(\mathrm{A})$ to $(\mathrm{F})$, with the seed solution, the potential, the orthogonality weight function and the "degree" of the extra member, that is the " $n$ " of the extra member $\mathcal{L}_{n}$. Case $(\mathrm{C})$ is omitted because it is identical with (B).

Summary table

\begin{tabular}{|c|c|c|c|c|}
\hline & seed & potential & weight & extra "deg." \\
\hline (A) & $e^{x^{2}}\left(3+4 x^{2}\right)^{3}$ & $x^{2}-\frac{3}{16 x^{2}}+\frac{48\left(-3+4 x^{2}\right)}{\left(3+4 x^{2}\right)^{2}}-\frac{13}{2}$ & $\frac{e^{-\eta} \eta^{1 / 4}}{(3+4 \eta)^{4}}$ & -2 \\
\hline (B) & $e^{x^{2}}\left(3+4 x^{2}\right)^{3}$ & $U_{A}+1$ & $\frac{e^{-\eta} \eta^{-1 / 4}}{(3+4 \eta)^{4}}$ & -3 \\
\hline (D) & $x^{-3 / 2}\left(-3+4 x^{2}\right)^{3}$ & $x^{2}-\frac{3}{16 x^{2}}+\frac{48\left(-9+4 x^{2}\right)}{\left(-3+4 x^{2}\right)^{2}}-\frac{11}{2}$ & n.a. & -2 \\
\hline (E) & $\left(x^{2}+6\right)^{3}\left(x^{2}+14\right)$ & $x^{2}+\frac{195}{4 x^{2}}+\frac{12\left(x^{2}-6\right)}{\left(x^{2}+6\right)^{2}}+\frac{4\left(x^{2}-14\right)}{\left(x^{2}+14\right)^{2}}-16$ & $\frac{e^{-\eta} \eta^{7}}{(6+\eta)^{4}(14+\eta)^{2}}$ & none \\
\hline (F) & $\left(x^{2}-6\right)^{3}\left(x^{2}-14\right)$ & $x^{2}+\frac{195}{4 x^{2}}+\frac{12\left(x^{2}+6\right)}{\left(x^{2}-6\right)^{2}}+\frac{4\left(x^{2}+14\right)}{\left(x^{2}-14\right)^{2}}+12$ & n.a. & none \\
\hline
\end{tabular}

\subsection{Three-index case}

Among various possibilities of the three index cases with lower degrees, the following has been noted to have a cubic zero:

$$
\mathrm{W}\left[\tilde{\phi}_{1}^{\mathrm{I}}\left(x ; \frac{53}{2}\right), \tilde{\phi}_{1}^{\mathrm{II}}\left(x ; \frac{53}{2}\right), \tilde{\phi}_{2}^{\mathrm{II}}\left(x ; \frac{53}{2}\right)\right](x)=-4 \frac{e^{-x^{2} / 2}}{x^{51 / 2}}\left(30+x^{2}\right)^{3}\left(390+39 x^{2}+x^{4}\right) .
$$


This case is denoted by $(\mathrm{H})$. The potential is

$$
U_{H}(x)=x^{2}+\frac{2499}{4 x^{2}}-52-\frac{720}{\left(30+x^{2}\right)^{2}}+\frac{12}{30+x^{2}}-\frac{312 x^{2}}{\left(390+39 x^{2}+x^{4}\right)^{2}}+\frac{4\left(2 x^{2}-39\right)}{390+39 x^{2}+x^{4}} .
$$

The global wavefunction of the Schrödinger equation with the eigenvalue $\mathcal{E}_{n}=4 n$ is

$$
\phi_{n}^{H}(x)=\frac{e^{-\eta / 2} \eta^{51 / 4}}{(30+\eta)^{2}\left(390+39 \eta+\eta^{2}\right)} \mathcal{L}_{n}^{H}(\eta), \quad n=0,1, \ldots
$$

in which $\mathcal{L}_{n}^{H}(\eta)$ is a degree $n+4$ polynomial in $\eta$. This belongs to the generic case, and the groundstate corresponds to $\mathcal{L}_{0}^{H}(\eta)$, which is proportional to $425880+67704 \eta+4004 \eta^{2}+$ $104 \eta^{3}+\eta^{4}$. The polynomial $\mathcal{L}_{n}^{H}(\eta)$ also satisfies a second-order differential equation with regular singularities at $\eta=0,-30,-39 / 2 \pm \sqrt{-39} / 2$ and an irregular singularity at $x=\infty$. The orthogonality measure for the polynomials $\left\{\mathcal{L}_{n}^{H}(\eta)\right\}$ is given by $\frac{e^{-\eta} \eta^{25} d \eta}{2(30+\eta)^{4}\left(390+39 \eta+\eta^{2}\right)^{2}}$.

At present we do not have a systematic means to locate seed solutions with high multiplicity of zeros, we stop our investigation here.

\section{Summary and comments}

Several families of infinitely many global solutions are presented for certain second-order differential equations (3.18), (3.23), (3.26), (3.30) and (3.35) having two or more finite regular singularities and one irregular singularity at infinity. The characteristic exponent of the global solutions at each finite singularity is $-2,-1$ or 0 . They are obtained from the non-generic as well as the generic two-indexed Laguerre polynomials by choosing the parameter $g$ in such a way that the seed solutions have a triple zero. In two families (D) and (F), the extra finite singularity is located inside the domain of the radial oscillator potential $(0, \infty)$. In the other three families, the extra singularity is outside of $(0, \infty)$ and thus they form orthogonal polynomials over $(0, \infty)$ with the weight functions (A) $W_{A}(\eta)=e^{-\eta} \eta^{1 / 4} /(3+4 \eta)^{4}$, (B) $W_{B}(\eta)=e^{-\eta} \eta^{-1 / 4} /(3+4 \eta)^{4}$, (E) $W_{E}(\eta)=e^{-\eta} \eta^{7} /\left\{(6+\eta)^{4}(14+\eta)^{2}\right\}$. Since they are obtained within the framework of multi-indexed orthogonal polynomials, their degrees have "gaps". Those polynomials belonging to $(\mathrm{A})$ and $(\mathrm{B})$ were derived in a different way in [9].

By construction, these families of two-indexed Laguerre polynomials are also the main part of eigenfunctions of exactly solvable quantum mechanics, belonging to the class called deformed radial oscillator potentials. In contrast with the generic multi-indexed polynomials $(\mathrm{E})$ and $(\mathrm{G})$, the systems obtained from the non-generic multi-indexed polynomials (A) and (B) are not shape invariant. By the standard Darboux-Crum transformations, these non-generic two-indexed polynomial systems are mapped to the generic one-indexed polynomial systems.

By using the same method, we tried in vain to find other global solutions with higher degrees $(\rho=-3,-4)$ of apparent singularities within the multi-indexed Laguerre polynomials. It would be interesting to explore the Jacobi polynomials counterparts of those results established in the present paper [12]. We wonder if the ideas of the present paper could be generalised to a certain kind of difference equations in discrete quantum mechanics $[6,16,17,18,22,23,27,28]^{2}$. The multi-indexed orthogonal polynomials are also established in the framework of difference Schrödinger equations, the multi-indexed $(q-)$ Racah, Wilson and Askey-Wilson polynomials [20, 21, 24, 25, 26].

\footnotetext{
${ }^{2}$ The dual $q$-Meixner polynomial in $[27, \S 5.2 .4]$ and dual $q$-Charlier polynomial in $[27, \S 5.2 .8]$ should be deleted because the hermiticity of the Hamiltonian is lost for these two cases.
} 


\section{Acknowledgements}

R.S. is supported in part by Grant-in-Aid for Scientific Research from the Ministry of Education, Culture, Sports, Science and Technology (MEXT), No.23540303 and No.22540186. K.T. is supported in part by the Grant-in-Aid for Young Scientists from the Japan Society for the Promotion of Science (JSPS), No.22740107.

\section{References}

[1] Adler V.È., On a modification of Crum's method, Theoret. and Math. Phys. 101 (1994), 1381-1386.

[2] Cooper F., Khare A., Sukhatme U., Supersymmetry and quantum mechanics, Phys. Rep. 251 (1995), 267385, hep-th/9405029.

[3] Crum M.M., Associated Sturm-Liouville systems, Quart. J. Math. Oxford Ser. (2) 6 (1955), 121-127, physics/9908019.

[4] Darboux G., Leçons sur la théorie générale des surfaces et les applications géométriques du calcul infinitésimal. II, Gauthier-Villars, Paris, 1989.

[5] Duistermaat J.J., Grünbaum F.A., Differential equations in the spectral parameter, Comm. Math. Phys. 103 (1986), 177-240.

[6] García-Gutiérrez L., Odake S., Sasaki R., Modification of Crum's theorem for 'discrete' quantum mechanics, Progr. Theoret. Phys. 124 (2010), 1-26, arXiv:1004.0289.

[7] Gendenshtein L.E., Derivation of exact spectra of the Schrödinger equation by means of supersymmetry, JETP Lett. 38 (1983), 356-359.

[8] Gibbons J., Veselov A.P., On the rational monodromy-free potentials with sextic growth, J. Math. Phys. 50 (2009), 013513, 25 pages, arXiv:0807.3502.

[9] Gómez-Ullate D., Kamran N., Milson R., A conjecture on exceptional orthogonal polynomials, Found. Comput. Math., to appear, arXiv:1203.6857.

[10] Gómez-Ullate D., Kamran N., Milson R., Two-step Darboux transformations and exceptional Laguerre polynomials, J. Math. Anal. Appl. 387 (2012), 410-418, arXiv:1103.5724.

[11] Hiroe K., Oshima T., A classification of roots of symmetric Kac-Moody root systems and its application, available at http://akagi.ms.u-tokyo.ac.jp/ oshima/index.html.

[12] Ho C.-L., Sasaki R., Takemura K., Confluence of apparent singularities in multi-indexed orthogonal polynomials: the Jacobi case, arXiv:1210.0207.

[13] Infeld L., Hull T.E., The factorization method, Rev. Modern Physics 23 (1951), 21-68.

[14] Krein M.G., On a continual analogue of a Christoffel formula from the theory of orthogonal polynomials, Dokl. Akad. Nauk SSSR 113 (1957), 970-973.

[15] Oblomkov A.A., Monodromy-free Schrödinger operators with quadratically increasing potential, Theoret. and Math. Phys. 121 (1999), 1574-1584.

[16] Odake S., Sasaki R., Crum's theorem for 'discrete' quantum mechanics, Progr. Theoret. Phys. 122 (2009), 1067-1079, arXiv:0902.2593.

[17] Odake S., Sasaki R., Exact solutions in the Heisenberg picture and annihilation-creation operators, Phys. Lett. B 641 (2006), 112-117, quant-ph/0605221.

[18] Odake S., Sasaki R., Exactly solvable 'discrete' quantum mechanics; shape invariance, Heisenberg solutions, annihilation-creation operators and coherent states, Progr. Theoret. Phys. 119 (2008), 663-700, arXiv:0802.1075.

[19] Odake S., Sasaki R., Exactly solvable quantum mechanics and infinite families of multi-indexed orthogonal polynomials, Phys. Lett. B 702 (2011), 164-170, arXiv:1105.0508.

[20] Odake S., Sasaki R., Exceptional Askey-Wilson-type polynomials through Darboux-Crum transformations, J. Phys. A: Math. Theor. 43 (2010), 335201, 18 pages, arXiv:1004.0544.

[21] Odake S., Sasaki R., Exceptional $\left(X_{\ell}\right)(q)$-Racah polynomials, Progr. Theoret. Phys. 125 (2011), 851-870, arXiv:1102.0813.

[22] Odake S., Sasaki R., Discrete quantum mechanics, J. Phys. A: Math. Theor. 44 (2011), 353001, 47 pages, arXiv:1104.0473. 
[23] Odake S., Sasaki R., Dual Christoffel transformations, Progr. Theoret. Phys. 126 (2011), 1-34, arXiv:1101.5468.

[24] Odake S., Sasaki R., Infinitely many shape invariant discrete quantum mechanical systems and new exceptional orthogonal polynomials related to the Wilson and Askey-Wilson polynomials, Phys. Lett. B 682 (2009), 130-136, arXiv:0909.3668.

[25] Odake S., Sasaki R., Multi-indexed (q-)Racah polynomials, J. Phys. A: Math. Theor. 45 (2012), 385201 , 21 pages, arXiv:1203.5868.

[26] Odake S., Sasaki R., Multi-indexed Wilson and Askey-Wilson polynomials, arXiv:1207.5584.

[27] Odake S., Sasaki R., Orthogonal polynomials from Hermitian matrices, J. Math. Phys. 49 (2008), 053503, 43 pages, arXiv:0712.4106.

[28] Odake S., Sasaki R., Unified theory of annihilation-creation operators for solvable ("discrete") quantum mechanics, J. Math. Phys. 47 (2006), 102102, 33 pages, quant-ph/0605215.

[29] Oshima T., Classification of Fuchsian systems and their connection problem, arXiv:0811.2916. 